\title{
HVCs, infall and the Galactic Fountain
}

\author{
Bart P. Wakker \\ supported by NASA/NSF; affiliated with Department of Astronomy \\ University of Wisconsin-Madison \\ 475 N Charter St, Madison, WI 53705, USA \\ email: wakker@astro.wisc.edu
}

\begin{abstract}
High-velocity clouds (HVCs) consist of gas that does not take part in normal Galactic rotation, having velocities deviating by up to $400 \mathrm{~km} \mathrm{~s}^{-1}$ from those expected from rotation. Over the past five decades, studies have shown they these clouds trace a number of different processes, including the Galactic Fountain, tidal streams, and infall. Here, I summarize some recent results concerning measurements of cloud metallicities and distances and how these are used to understand individual clouds and derive the conclusion above.
\end{abstract}

Keywords. ISM: general, ISM: HVCs, Galaxy: corona

Observations using the ultraviolet spectrographs on the Hubble Space Telescope (HST), as well as with the Far Ultraviolet Spectroscopic Explorer (FUSE) have now yielded measurements of the S II $\lambda \lambda 1250,1253,1259$ and many O I lines. Because the ionization potentials of $\mathrm{OI}$ and $\mathrm{HI}$ are similar and because most of the oxygen is in the gas phase, the O I/H I ratio is usually the same as the O/H ratio. Similarly, SII/H I is usually close to $\mathrm{S} / \mathrm{H}$, although sometimes a (small) ionization correction is needed.

Most published results so far concentrate on measurements toward HVC complex C (see Fox et al. 2004 for a summary) and the Magellanic Stream (Gibson et al. 2000; Fox et al. 2005). From our HST Cycle 18 program, as well as an analysis of the FUSE+HST archive we now have 17 different clouds toward which the metallicity of a HVC has been measured, with values ranging from $1 / 20$ th solar to solar. There is no exact correlation between velocity and metallicity. Values of $1 / 20$ th, $1 / 10$ th and 1 times solar are found for intermediate-velocity clouds (HVCs with $|v|<90 \mathrm{~km} \mathrm{~s}^{-1}$ ); values ranging from 1/10th to $1 / 4$ solar are found in the Magellanic Stream; while for clouds with $|v|<90 \mathrm{~km} \mathrm{~s}^{-1}$ the metallicities range from $1 / 10$ th solar to solar. The cloud metallicities allow us to associate individual clouds with one of the main processes summarized above.

To find distances to HVCs requires a set of stellar targets situated both behind and in front of the star. Such targets need to have clean spectra and known distance. Starting with the SDSS and 2MASS surveys, we defined a sample of 3000 candidate probes toward $60 \mathrm{HVCs}$. We now have good distances for some $15 \mathrm{HVCs}$, using intermediate-resolution (1 $\AA$ ) spectra from the SDSS ( $\sim 800$ stars), spectra taken by us using the APO $(\sim 1200$ stars), photometry from the SDSS ( $\sim 500$ stars), our own photometry program on the WIYN $0.9 \mathrm{~m}$ (160 nights, $\sim 100$ stars $)$, as well as high-resolution $\left(\sim 6 \mathrm{~km}^{-1}\right)$ spectra for 125 stars taken with Keck/HIRES and the VLT/UVES. These distances reveal that the lower-velocity clouds $\left(|v|<90 \mathrm{~km} \mathrm{~s}^{-1}\right)$ tend to be located up $\sim 1-5 \mathrm{kpc}$ above the Galactic disk, while higher-velocity clouds range in distance from 3 to $25 \mathrm{kpc}$ (some of these results were shown in Wakker et al. $(2007,2008)$. The three most massive HVCs for which we determined a distance are about $7 \mathrm{kpc}$ above the plane. Fig. 1 shows an image of the Milky Way, with the locations of the HVCs with known distances overlaid. 


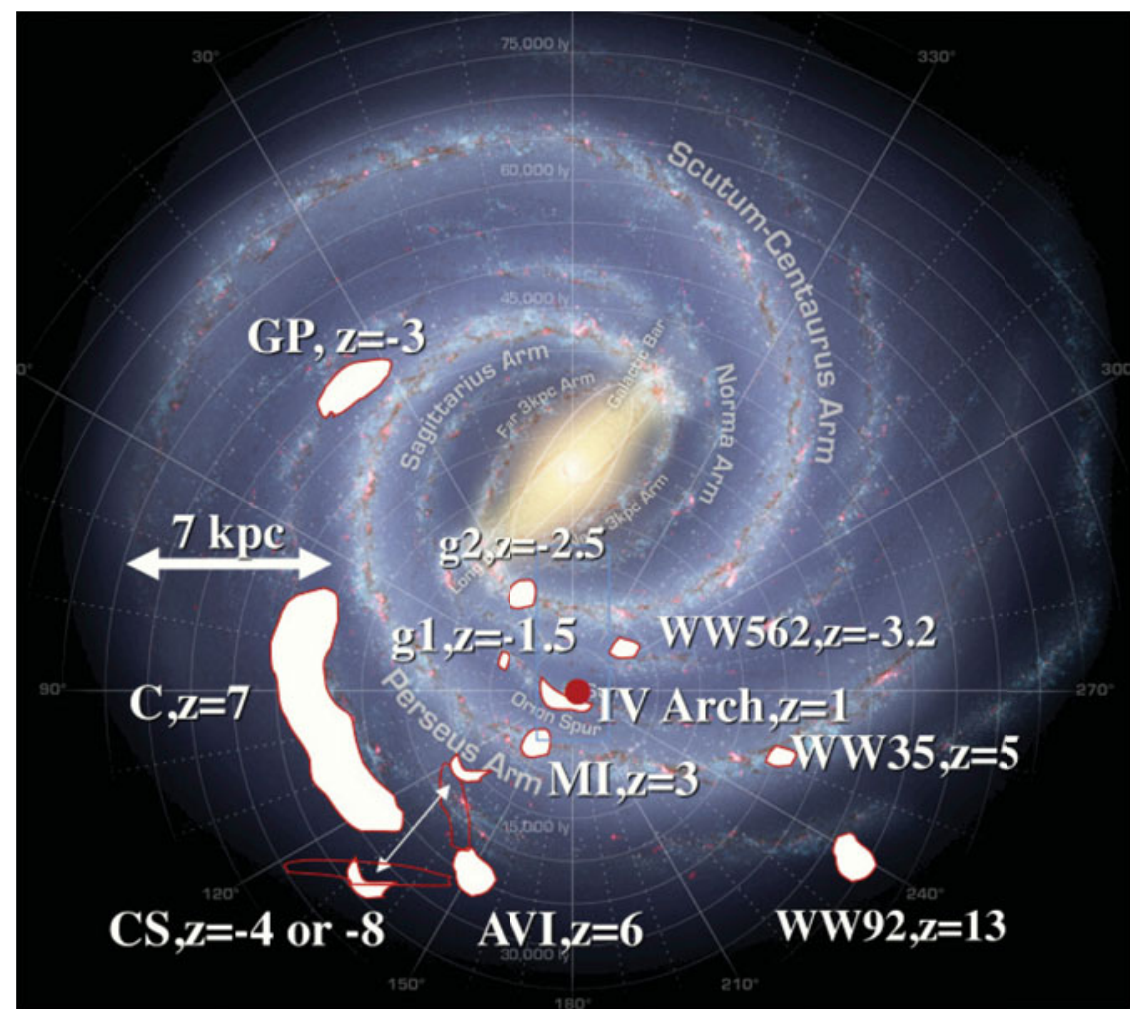

Figure 1. Artist impression image of the Milky Way, with the locations of the HVCs with known distances overlaid

\section{References}

Fox, A., Savage, B., Wakker, B., et al. 2004, ApJ 602, 738

Fox, A., Wakker, B., Smoker, J., et al. 2010, ApJ 718, 1046

Gibson, B., Giroux, M., Penton, S., et al. 2000, AJ 120, 1830

Wakker, B., York, D., Howk, J., et al. 2007, ApJ (Letters) 670, L113

Wakker, B., York, D., Wilhelm, R., et al. 2008, ApJ, 672, 298 